\title{
The Prevalence and Nature of Cancer-Related Neuropathic Pain in Out-Patient Services in Spain
}

\author{
Cristina Garzón-Rodríguez ${ }^{1, *}$, Luis Olay Gayoso ${ }^{2}$, Juan M. Sepulveda $^{3}$, Enrique Martínez ${ }^{4}$, \\ Ignacio Peláez ${ }^{5}$, Luis de la Cruz Merino ${ }^{6}$, Gary Atkinson ${ }^{7}$, Vanessa López-Gómez ${ }^{8}$, \\ Maria Pérez Páramo ${ }^{8}$ and Chantal van Litsenburg ${ }^{9}$
}

${ }^{1}$ Service of Palliative Care, Institut Catala d'Oncologia. Barcelona, Spain; ${ }^{2}$ Medical Oncology Radiotherapy Unit in Huca Oviedo, Gijón y alrededores, Spain; ${ }^{3}$ Medical Oncology, Hospital Universitario 12 de Octubre, Madrid, Spain; ${ }^{4}$ Hospital de Navarra, Navarra, Spain; ${ }^{5}$ Hospital de Cabueñes, Gijón, Asturias, Spain; ${ }^{6}$ Hospital Universitario Virgen Macarena, Seville, Spain; ${ }^{7}$ Pfizer Ltd., Walton Oaks, UK; ${ }^{8}$ Pfizer, Medical Unit, Madrid, Spain; ${ }^{9}$ Pfizer bv, Capelle a/d IJssel, The Netherlands

\begin{abstract}
Cancer patients with neuropathic pain have been shown to have higher levels of pain interference and impaired quality of life (QoL) compared with cancer patients without neuropathic pain. This article presents sub-analysis of data from a large non-interventional study conducted between 23 August 2010 and 22 July 2011 in Denmark, Germany, Greece, Spain and the UK. Descriptive data collected in 21 oncology or palliative care centers during patients' routine out-patient visits in Spain are reported here to further investigate the prevalence and nature of cancer-related neuropathic pain (CRNP). The prevalence of CRNP in 557 cancer patients with chronic pain in Spain was estimated at $30.9 \%(95 \%$ confidence interval [CI]: 27.0-34.7) by physicians before considering scores on the PainDETECT questionnaire and 30.7\% (95\% CI: 26.9-34.5) after considering PainDETECT scores. Despite a similar overall number, there were shifts in the numbers of patients with or without a diagnosis of CRNP after physicians had had the opportunity to consider PainDETECT scores, particularly for patients who received an initial diagnosis of 'unknown'. In the opinion of the physicians, 92/171 (53.8\%) of the CRNP population had mixed cancer-related pain with a neuropathic component, which can be difficult to identify and effectively manage. The self-reported responses to questions and scales in this Spanish sample indicated that many aspects of daily functioning were negatively impacted within the CRNP participants and that pain was not always controlled. These data highlight the need for national guidelines to identify and treat cancer-related pain with a neuropathic component.
\end{abstract}

Keywords: Clinical oncology, neuropathic pain, outpatients, quality of life, questionnaire.

\section{INTRODUCTION}

Based on statistical models of available data from 40 countries in 2008, it was estimated that there are approximately 3.2 million new cases of cancer each year in Europe [1]. The age-standardized incidence-rates in Spain were estimated at 253.6 cases per 100,000 person-years for women (most commonly breast cancer) and 450.3 cases per 100,000 person-years for men (most commonly prostate cancer) [1].

Cancer-related pain has been estimated to affect half of all cancer patients, on average, and is more prevalent in advanced disease than in the early stages [2]. Neuropathic pain (as opposed to nociceptive pain) can result from damage to the peripheral or the central nervous system resulting in hypersensitivity to pain [3]. Cancer patients with neuropathic pain have been shown to have higher levels of pain interference and impaired quality of life (QoL) (many

*Address correspondence to this author at the Servicio de Cuidados Paliativos, Institut Catalá d'Oncologia, Avda Gran Via de L'Hospitalet 199-203, 08908 L'Hospitalet de Llobregat, Barcelona, Spain;

Tel: +34 93 2607789; Fax: +34 93 2607829;

E-mail: cgrodriguez@iconcologia.net experiencing physical and/or psychological disability) compared with cancer patients without neuropathic pain [4].

Up to $40 \%$ of cancer patients with pain are estimated to experience pain with a neuropathic component [5]. In particular, lung and breast cancer have both been associated with neuropathic pain [6]. In Spain, the prevalence of neuropathic pain among cancer patients was estimated at approximately $31 \%$ to $33 \%$ in patients experiencing pain visiting either radiotherapy oncology units [6] or oncology clinics [7], using the Douleur Neuropathique 4 questions (DN4) tool [8]. Other tools are also available to help in the diagnosis of neuropathic pain [9], but none are specifically validated in the cancer population.

The prevalence of cancer-related neuropathic pain (CRNP) in out-patients experiencing chronic pain (regardless of stage or type of cancer) was estimated at $32.6 \%$ in a noninterventional, cross-sectional multicenter study conducted in 5 countries within Europe (Denmark, Germany, Greece, Spain and the UK) [10]. To further investigate the prevalence of neuropathic pain in an out-patient sample of cancer patients in Spain, outcomes of a sub-analysis of these data are reported within this article. Further aims of the study 
were to assess the characteristics and nature of the pain and the QoL within the participants with CRNP. The usefulness of the PainDETECT questionnaire [11], which has been adapted for use in Spain [12], as a tool to help physicians identify neuropathic pain in cancer patients was also assessed.

\section{MATERIALS AND METHODOLOGY}

\section{Study Design}

The original non-interventional study was conducted between 23 August 2010 and 22 July 2011 in out-patient clinics in Denmark, Germany, Greece, Spain and the UK. A sub-analysis of data collected in 21 oncology or palliative care centers during patients' routine out-patient visits in Spain is reported here. To minimize a potential recruitment bias, patients were consecutively identified for eligibility (i.e. each patient attending the clinic during the recruitment period was considered for participation in the study) (Fig. 1). Institutional Review Boards/Independent Ethics Committees at participating sites reviewed and approved the study protocol.

\section{Participants}

All participants were adults aged $\geq 18$ years, had chronic pain (pain for most days of the week for $\geq 3$ months) and consulted with a physician at an out-patient clinic (oncology or palliative care) for any reason and at any stage of the cancer. Patients were excluded from the study if their chronic pain was considered by the physician to be unrelated to the cancer. All enrolled participants provided informed consent to participate in the study.

\section{Study Enrollment and Procedures}

The PainDETECT neuropathic pain screening tool was used in conjunction with the physician assessment to identify patients with chronic pain who also had CRNP. For all patients that were considered to have CRNP following clinical assessment of the PainDETECT questionnaire, casereport forms (CRFs) were completed by participants and their physicians. The responses to the CRFs, in addition to other self-reported questionnaires, were used to assess the following:

- Severity and interference of the pain using the Modified Brief Pain Inventory Short Form (m-BPI-sf) [13].

- History and clinical management of the cancer (type of therapeutic intervention since time of diagnosis) and the neuropathic pain (duration, etiology, pharmacological treatment/s for pain etc.).

- Health-related QoL using the EQ-5D Health Questionnaire (EuroQoL) (mobility, self-care, usual activities, pain/discomfort, anxiety and depression were rated on a 3-point scale and combined into an index utility score on a scale of 0-1) [14]. In addition, participants' perception of their own health was also assessed on a numerical rating scale (0-100).

- Functional impairment relating to home life/family responsibilities work/school and social life was assessed using The Sheehan Disability Scale (SDS) (max score of $30=$ highly impaired) [8].

\section{Outcome Measures}

The primary endpoint of this sub-analysis was the proportion of patients with CRNP (as defined by the clinician after reviewing the outcomes of the PainDETECT questionnaire) out of the total number of patients with chronic pain who attended out-patient clinics in Spain.

Secondary endpoints included:

- CRNP participants' own assessment of the disease and pain and the impact on daily life.

- Physicians' assessment of the characteristics and the pharmacological management of the pain and the cancer in the CRNP population.

- The percentage of physicians who completed the physician-specific CRFs that found the screening tool a useful instrument to identify chronic neuropathic pain in daily practice (based on physician responses regarding the usefulness of the tool).

\section{Statistical Analysis}

\section{Sample size and Study Populations}

It was pre-planned that the enrollment of at least 200 patients in Spain would achieve an estimated precision of 0.07 (7\%).

Study populations in this study were defined as 'All participants' (all those that enrolled in the study), 'CRNP population' (those patients diagnosed with CRNP after examining scores on the PainDETECT questionnaire), and 'Surveyed-physicians' population (physicians who completed some, or all of the physician-specific CRF following completion of the study).

\section{Data Analysis}

For discrete endpoints, frequency and percentage calculations were used for each response category (any missing data were excluded from these calculations). Continuous endpoints were summarized descriptively. 95\% confidence intervals for continuous variables were calculated using the normal distribution, and for categorical endpoints the normal approximation to the binomial distribution was used. All calculations were performed using SAS $^{\circledR}$ Version 9.2 (SAS Institute, Cary, NC, USA).

\section{RESULTS}

\section{Study Populations and Demographics}

In total, 557 patients with chronic pain were enrolled in the study in Spain ('All participant' population) and 552 participants $(99.1 \%)$ completed all or part of the study. There were missing data (either CRF page or final status missing) for $5(0.9 \%)$ participants. A total of 36 physicians enrolled participants for the study and 22 completed the physicianspecific CRF. The mean age of the overall study population was 63 years (Table 1). There were 324 males and 222 females and 6 participants for whom sex was not recorded.

172 of the 557 participants enrolled in the study $(30.9 \%$; $95 \%$ confidence interval [CI]: 27.0-34.7) were identified to have CRNP in the clinical opinion of the physicians before the use of the PainDETECT screening tool. A similar 


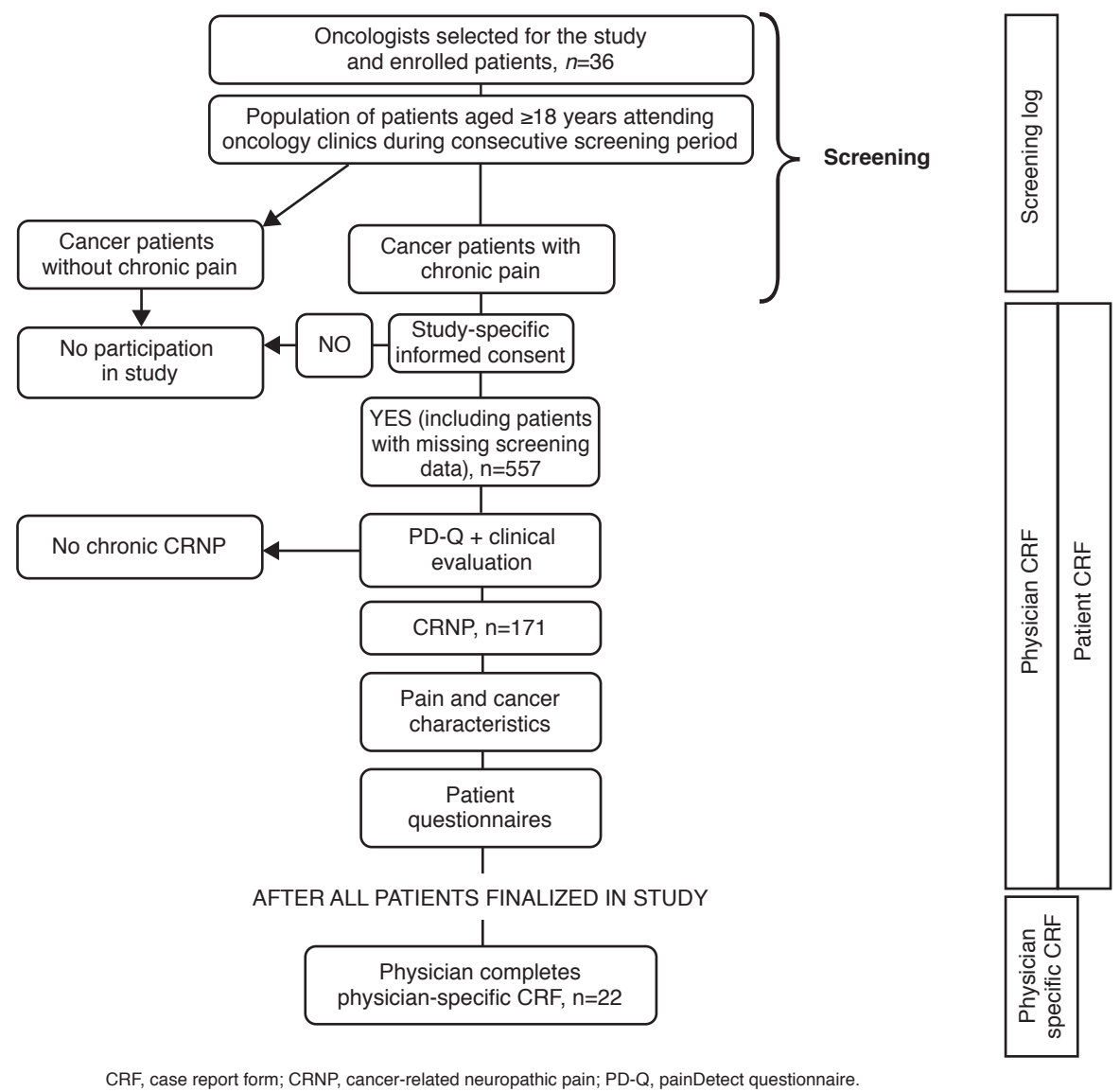

Fig. (1). Procedure flow. CRF, case report form; CRNP, cancer-related neuropathic pain; PD-Q, painDetect questionnaire.

Table 1. Age and Sex of Patient Populations for All Participants and CRNP Population

\begin{tabular}{|c|c|c|c|c|}
\hline & Male & Female & Participants for Whom Sex was Not Recorded & Total \\
\hline All Participants ${ }^{\mathrm{a}}, \mathrm{N}$ & 324 & 222 & 7 & 553 \\
\hline $18-44$ & $11(3.4)$ & $19(8.6)$ & $2(28.6)$ & $32(5.8)$ \\
\hline $45-64$ & $142(43.8)$ & $122(55.0)$ & $2(28.6)$ & $266(48.1)$ \\
\hline Missing Data & 0 & 0 & $2(28.6)$ & $2(0.4)$ \\
\hline Mean (SD) & $64.8(11.5)$ & $60.4(12.5)$ & $51.2(17.0)$ & $62.9(12.2)$ \\
\hline Patients With $\mathrm{CRNP}^{\mathrm{b}}, \mathrm{N}$ & 91 & 72 & 7 & 170 \\
\hline$\geq 65$ & $42(46.2)$ & $22(30.6)$ & $1(14.3)$ & $65(38.2)$ \\
\hline Missing Data & 0 & 0 & $2(28.6)$ & $2(1.2)$ \\
\hline Mean (SD) & $62.8(10.7)$ & $57.8(12.5)$ & $51.2(17.0)$ & $60.3(12.0)$ \\
\hline
\end{tabular}

a Incorrect dates of birth were recorded for 4 patients in the 'All participant' population and excluded from the demographic summary.

bAge data are missing for 1 patient in the 'Neuropathic pain' population.

CRNP, cancer-related neuropathic pain. 
number of participants $(171 ; 30.7 \%$; 95\% CI: 26.9-34.5) were considered to have CRNP after the use of PainDETECT tool. The demographic characteristics (age and sex) of the CRNP participants are shown in Table 1.

Type and Therapeutic Management of Cancer in CRNP Participants

\section{Types and Characteristics of Cancer}

The most common types of cancer within the CRNP population were lung cancer $(19.9 \%)$ and colorectal cancer (18.1\%) (Table 2). Half of all CRNP participants (50.9\%) had loco-regional progression of the cancer.

\section{Summary of Therapeutic Management of Cancer}

Of the 557 CRNP participants, 117 (68.4\%) received prior or ongoing chemotherapy and 95 CRNP participants $(55.6 \%)$ received prior or ongoing radiotherapy treatment (Table 3). Some of these may have received both chemotherapy and radiotherapy treatment.

\section{Summary of Recorded Surgical Treatment for Cancer}

Surgical procedures in CRNP participants included exeresis, colectomy/colostomy, hysterectomy/ hysterosalpingo-oophorectomy, laparotomy, lymphadenectomy and mastectomy. The surgical procedures were categorized into treatment types: neoadjuvant (cancer treatment prior to surgery), adjuvant (cancer treatment following surgery), advanced/metastatic (surgical removal of body parts), palliative (to relieve symptoms) and are summarized in Table 3. The surgical category data were missing for 18 participants $(10.5 \%)$ and the surgery CRF page was missing for $15(8.8 \%)$ participants.

Type and Therapeutic Management of Pain in CRNP Participants

Type and Cause of Neuropathic Pain Based on Physicians' Assessments

In the opinion of the physicians, 60/171 (35.1\%) of the CRNP participants had neuropathic-type pain and 92/171 $(53.8 \%)$ had mixed cancer-related pain with a neuropathic component. Data were missing for 19/171 (11.1\%) participants. The neuropathic pain experienced by CRNP participants was considered to be due to the tumor itself in most cases $(124 / 171 ; 72.5 \%)$, and due to cancer treatment in $55 / 171(32.2 \%)$ cases. Data were missing for 15 participants $(8.8 \%)$. More than 1 response was possible for the relationship between CRNP and type of cancer treatment. However, the majority of treatment-related CRNP was attributed to chemotherapy (16.4\%) and/or surgery (14.6\%).

\section{Duration of Neuropathic Pain}

22 of the CRNP participants had experienced CRNP for $<3$ months $(12.9 \%) ; 73$ participants $(42.7 \%)$ for 3 to 6 months; 34 participants $(19.9 \%)$ for 7 to 12 months, 23 $(13.5 \%)$ for 13 months to 3 years and $4(2.3 \%)$ for $>3$ years. Data were missing for 15 (8.8\%) participants.

\section{Pain-Related Characteristics of CRNP: mBPI-sf}

Mean subscale scores on the m-BPI-sf in this population were 5.6 ( $95 \%$ CI: $5.2-5.9 ; \mathrm{n}=155)$ for 'Interference' and 4.8 (95\% CI: 4.4-5.1; $\mathrm{n}=158)$ for 'Severity' based on numerical rating scales of 0 (no pain) to 10 (pain as bad as you can imagine). Data were missing for 16 participants and 13 participants for interference and severity, respectively.

Table 2. Summary of Characteristics and Type of Cancer in CRNP Patients

\begin{tabular}{|c|c|}
\hline Characteristics of Cancer & $N=171$ \\
\hline \multicolumn{2}{|l|}{ Cancer Type ${ }^{a}$, n (\%) } \\
\hline Breast & $25(14.62)$ \\
\hline Lung & $34(19.88)$ \\
\hline Prostate & $17(9.94)$ \\
\hline Colorectal & $31(18.13)$ \\
\hline Other $^{b}$ & $54(31.58)$ \\
\hline Missing Data & $13(7.60)$ \\
\hline \multicolumn{2}{|l|}{$\begin{array}{c}\text { Loco-Regional Progression of the } \\
\text { Cancer, } \mathrm{n}(\%)\end{array}$} \\
\hline Yes & $87(50.88)$ \\
\hline No & $65(38.01)$ \\
\hline Missing Data & $19(11.11)$ \\
\hline \multicolumn{2}{|l|}{ Sites of Metastasis, n (\%) } \\
\hline Brain & $6(3.51)$ \\
\hline Bone & $70(40.94)$ \\
\hline Lung & $41(23.98)$ \\
\hline Lymph Node & $48(28.07)$ \\
\hline Liver & $23(13.45)$ \\
\hline Other & $20(11.70)$ \\
\hline Missing Data & $55(32.16)$ \\
\hline \multicolumn{2}{|l|}{$\begin{array}{l}\text { Total Number of Sites of } \\
\text { Metastasis, } \mathrm{n}(\%)\end{array}$} \\
\hline 0 & $24(14.04)$ \\
\hline 1 & $35(20.47)$ \\
\hline 2 & $35(20.47)$ \\
\hline 3 & $12(7.02)$ \\
\hline 4 & $6(3.51)$ \\
\hline 5 & $2(1.17)$ \\
\hline 10 & $2(1.17)$ \\
\hline Missing Data & $55(32.16)$ \\
\hline
\end{tabular}

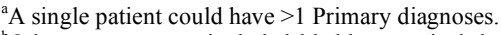

'Other cancer types included bladder, cervical, head and neck, lymphoma, myeloma, pancreatic as well as other MedDRA lowest level term types of cancer, including unknown primary cancer.

Mean scores for individual items on the m-BPI-sf for CRNP participants were all $>4.5$, with the exception of "pain 
right now', (4.1; 95\% CI: 3.7-4.5); and 'pain at its least' in the last 24 hours $(3.0 ; 95 \%$ CI: $2.7-3.4)$. The highest mean scores were for 'worst pain' in the last 24 hours $(6.9 ; 95 \%$ CI: 6.5-7.3); and for 'impact on normal work' $(6.6 ; 95 \% \mathrm{CI}$ : 6.1-7.1).

Table 3. Therapeutic Management and Surgical Treatment of Cancer in CRNP Patients

\begin{tabular}{|c|c|c|}
\hline \multirow[b]{2}{*}{ Therapy $^{a}$} & \multicolumn{2}{|c|}{$N=171$} \\
\hline & Yes & No \\
\hline Chemotherapy $^{\mathrm{b}}, \mathrm{n}(\%)$ & $117(68.42)$ & $36(21.05)$ \\
\hline Radiotherapy, n (\%) & $95(55.56)$ & $58(33.92)$ \\
\hline Prior & $74(43.27)$ & \\
\hline Ongoing & $16(9.36)$ & \\
\hline Endocrine (Hormone) Therapy, n (\%) & $36(21.05)$ & $91(53.22)$ \\
\hline Prior & $9(5.26)$ & \\
\hline Ongoing & $25(14.62)$ & \\
\hline Multi-Targeted Substances, n (\%) & $22(12.87)$ & $98(57.31)$ \\
\hline Prior & $7(4.09)$ & \\
\hline Ongoing & $12(7.02)$ & \\
\hline Other, n (\%) & $23(13.45)$ & $52(30.41)$ \\
\hline Prior & $6(3.51)$ & \\
\hline Ongoing & $15(8.77)$ & \\
\hline Recorded Surgery Treatment $^{\mathrm{a}}$ & & $59(34.50)$ \\
\hline Neoadjuvant & $31(18.13)$ & \\
\hline Adjuvant & $38(22.22)$ & \\
\hline Advanced/Metastatic & $16(9.36)$ & \\
\hline Palliative & $15(8.77)$ & \\
\hline
\end{tabular}

${ }^{a}$ More than 1 therapy or treatment type was possible for each patient.

${ }^{\mathrm{b}}$ Chemotherapy treatment included platinum compounds, vinca alkaloids, taxanes, antimetabolites and nitrosoureas among others. Treatment could have been prior to, or ongoing at the time of the study.

\section{Therapeutic Management of Neuropathic Pain}

The most common previously prescribed therapeutic treatments for neuropathic pain in CRNP participants were non-opioid analgesics and/or a strong opioid. For current treatment, anticonvulsants were the most commonly prescribed (Table 4).

\section{Participant Reported Outcomes: CRF Responses}

Over half of the CRNP population (92/171; 53.8\%) indicated that their symptoms had an effect on their employment status, $33.3 \%$ indicated that they did not. Data were missing for 22 participants (12.9\%). Approximately $28 \%(48 / 171)$ of the CRNP participants recorded that they had not visited their doctor over the past 4 weeks, $24.0 \%$ recorded 1 visit, $22.8 \%$ recorded 2 visits, $9.4 \%$ recorded 3 visits, and $8.2 \%$ recorded 4 or more visits. Data were missing for 13 participants (7.6\%). Only 5 participants $(2.9 \%)$ reported that they had not used treatment during the 4 weeks prior to the clinic visit, while 152 participants (88.9\%) reported that they had used prescription medications. Other reported treatments were as follows: non-prescription medications $=9$ participants $(5.3 \%)$; physiotherapy $=9$ participants $(5.3 \%)$; massage $=6$ participants $(3.5 \%)$; other treatments $=11$ participants $(6.4 \%)$. Participants could indicate more than one treatment type.

Table 4. Therapeutic Management of Neuropathic Pain in CRNP Patients

\begin{tabular}{|c|c|c|}
\hline $\begin{array}{c}\text { Prescribed Treatment for } \\
\text { Neuropathic Pain, } \mathbf{n}(\%)^{\mathbf{a}}\end{array}$ & $\begin{array}{c}\text { Previous } \\
\mathbf{N = 1 7 1}\end{array}$ & $\begin{array}{c}\text { Current } \\
\mathbf{N}=\mathbf{1 7 1}\end{array}$ \\
\hline \hline Non-Opioid Analgesics & $96(56.14)$ & $86(50.29)$ \\
\hline Weak Opioids & $19(11.11)$ & $8(4.68)$ \\
\hline Strong Opioids & $86(50.29)$ & $87(50.88)$ \\
\hline Antidepressants & $22(12.87)$ & $22(12.87)$ \\
\hline Anticonvulsants & $74(43.27)$ & $100(58.48)$ \\
\hline Muscle Relaxants & $9(5.26)$ & $6(3.51)$ \\
\hline Corticosteroids & $39(22.81)$ & $41(23.98)$ \\
\hline Antispasmodics & $2(1.17)$ & 0 \\
\hline Anxiolytics & $36(21.05)$ & $28(16.37)$ \\
\hline Other & $13(7.60)$ & $16(9.36)$ \\
\hline None & $4(2.34)$ & $6(3.51)$ \\
\hline
\end{tabular}

${ }^{\mathrm{a}}$ More than 1 treatment type was possible for each patient.

\section{CRNP Participant Reported QoL and Health Outcomes: EuroQoL}

Mean scores for CRNP participants were $50.2(95 \% \mathrm{CI}$ : 47.0-53.5) on a scale of $0-100$ for Health State, and 0.5 (95\% CI: 0.5-0.6) on a scale of $0-1$ for Utility (higher scores indicate better health). Data were missing for 15 participants and 17 participants for Health State, and Utility Scores, respectively.

\section{SDS}

The mean total score for global functional impairment on the SDS scale (range 0-30) for CRNP participants was 18.6 (95\% CI: 17.3-20.0) (higher scores indicate greater impairment). Data were missing for 38 participants (Table 5). CRNP participants' mean scores for the individual items on SDS (range 0-10) were highest in relation to the impact on work/school, family life, and social life (Table 5).

\section{Pain Assessment on PainDETECT}

Participant assessment of pain scores recorded in response to the preliminary questions on the PainDETECT questionnaire were higher, on average, for CRNP $(n=170)$ participants (data were missing for 1 participant) than for all participants $(n=557)$ (data were missing for up to 16 participants) in response to each of the following questions: 'How would you assess your pain now, at this moment?', 4.5 (95\% CI: 4.1-4.9) versus 3.9 (95\% CI: 3.7-4.2); 'How strong was the strongest pain during the past 4 weeks?', 8.1 (95\% 
Table 5. Summary of Individual Items and Total Score on SDS.

\begin{tabular}{|c|c|c|c|c|}
\hline & $\mathbf{n}$ & Mean (SD) & 95\% CI & Missing Data, n \\
\hline \hline Days Lost & 139 & $4.70(5.18)$ & $3.83,5.57$ & 32 \\
\hline Days Unproductive & 136 & $4.04(2.94)$ & $5.42,6.33$ & 14 \\
\hline Family Life & 157 & $5.87(2.89)$ & $5.27,6.21$ & 14 \\
\hline Social Life & 157 & $5.74(2.99)$ & $6.50,7.41$ & 38 \\
\hline Work/School & 133 & $6.95(2.68)$ & $17.30,19.96$ & 38 \\
\hline Total Score & 133 & $18.63(7.77)$ & \\
\hline
\end{tabular}

CI: 7.8-8.4) versus 7.6 (95\% CI: 7.5-7.8); and 'How strong was the pain during the past 4 weeks on average?', $5.5(95 \%$ CI: 5.2-5.8) versus $5.2(95 \%$ CI: 5.0-5.3) for CRNP participants versus all participants, respectively.

\section{Evaluation of PainDETECT Questionnaire (Physicians, Assessments)}

Over $80 \%(18 / 22)$ of the surveyed-physician population (i.e. those that completed the physician-specific CRF) indicated that they found the PainDETECT questionnaire useful and the remaining 4 physicians indicated that they did not. Physicians responded positively ('yes') to the question 'Did the PainDETECT questionnaire help you evaluate if you think the patient has CRNP?' in relation to $192 / 557$ (34.5\%) of participants. They responded 'no' for 334/557 $(60.0 \%)$ participants and data were missing for 31 participants $(5.6 \%)$. In a 5 item response (All/Most/Some/Few/None) to the question 'In future would you use the PainDETECT questionnaire?' none of the physicians responded 'yes' to ALL; however, 8/22 (36.4\%) responded 'yes' for MOST patients, 7/22 (31.8\%) for SOME patients and $4 / 22(18.2 \%)$ for FEW patients. 3/22 (13.6\%) physicians indicated that they would not use it.

\section{Impact of PainDETECT end Scores on Physicians, Clinical Assessment}

Of the participants diagnosed with CRNP, the greatest number had end scores of $>18$ (likely CRNP), fewer had end scores of 13-18 (possible CRNP), and the fewest had end scores of $<13$ (unlikely CRNP). For participants without CRNP, the opposite trend was shown (Fig. 2). There were more changes to a non-diagnosis of CRNP from an initial positive diagnosis than to a diagnosis of CRNP from an initial non-diagnosis following physicians' assessment of PainDETECT (Fig. 3). Most of the changes occurred in participants with end scores of $<13$, and whose previous diagnosis was recorded as 'unknown'.

\section{DISCUSSION}

The results of this sub-analysis of data collected from cancer patients visiting out-patient units in Spain are largely consistent with the results of the main study that included data from 5 European countries [10]. It should be noted, however, that nearly half of the patients screened for the main study (47\%) were attending clinics in Spain. Therefore, the Spanish population represented the largest proportion of participants included in the main study. The prevalence of
CRNP in the 951 cancer patients that attended clinics in one of the 5 European countries was estimated at $32.6 \%$ by physicians after considering scores on the PainDETECT questionnaire [10]. Based on the sub-sample of data from Spain, the Spanish CRNP population was estimated at $30.7 \%$. Due to the higher number of participating physicians than anticipated, the number of enrolled participants in this Spanish sample $(n=557)$ was much larger than the planned sub-analysis sample size of at least 200 patients, and therefore exceeds the estimated precision rate of $7 \%$.

The most common types of cancer in the CRNP participants were lung, colorectal and breast cancer, even though less than half of the CRNP participants were female. As lung and breast cancer have both been previously associated with neuropathic pain [6], a potentially increased likelihood of patients with lung and breast cancer experiencing neuropathic pain may warrant further consideration.

Self-reported pain scores in the CRNP participants was numerically higher, on average, than among the 'all participant' population, at least based on the preliminary PainDETECT questions. However, the study was not planned to conduct comparative statistical analyses between these populations as the CRNP participants were also a subgroup of the 'all participant' population.

Although $\sim 68 \%$ of the CRNP participants had prior or ongoing chemotherapy and/or $\sim 55 \%$ had prior or ongoing radiotherapy, physicians attributed the neuropathic pain to the tumor itself for the majority of CRNP participants $(\sim 73 \%)$, rather than the cancer treatment. This is consistent with findings of a prospective epidemiological study in which the tumor was considered to be the cause of CRNP in $75 \%$ of patients attending radiotherapy oncology units in Spain [6]. This was somewhat unexpected, as chemotherapyinduced peripheral neuropathy is a well-known subset of CRNP and its diagnosis is critical for providing appropriate treatment which sometimes requires stopping the chemotherapy treatment to allow recovery of the nerves [15]. Also, it was anticipated that there would be fewer patients with progressed disease within the population of outpatients included in this study than that seen within radiotherapy clinics. However, the average duration since diagnosis of cancer was not recorded in this European survey.

In our study, the most common previously prescribed therapeutic treatments for neuropathic pain in the CRNP participants were non-opioid analgesics and/or a strong 


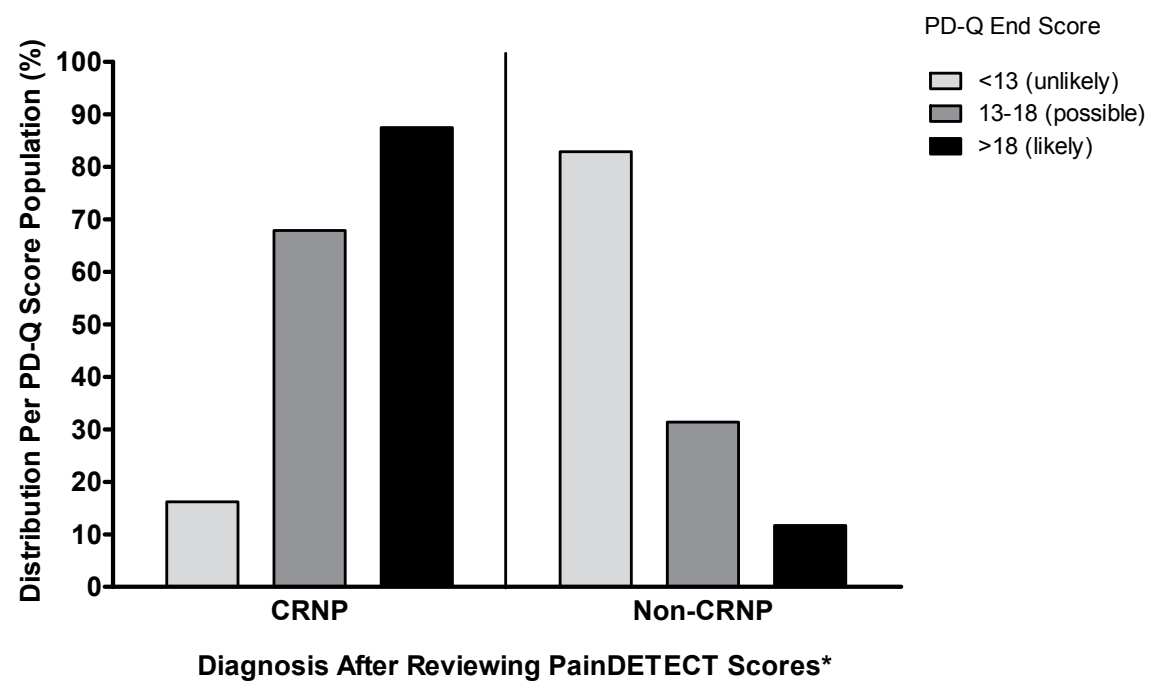

*PainDETECT scores and/or diagnosis were unavailable for 19 patients.

Fig. (2). Distribution of PainDETECT end scores for patients with or without a diagnosis of CRNP. PD-Q, PainDETECT questionnaire.

* PainDETECT scores and/or diagnosis were unavailable for 19, patients.

PD-Q End Score*

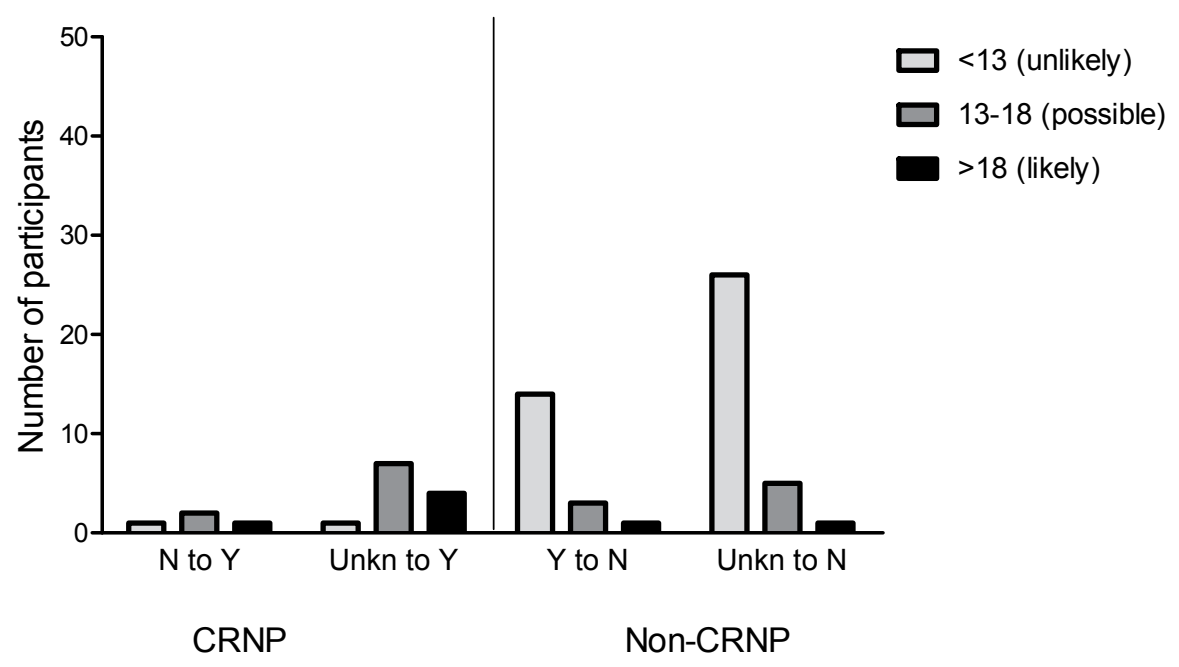

Fig. (3). Shifts in physicians' initial diagnosis of patients with or without CRNP (CRNP or non-CRNP) after examination of the PainDETECT questionnaire, distributed by end scores. *Data were missing for 19 patients. Missing data not shown. N, no; Y, yes; Unkn, unknown.

opioid. For current treatment, anticonvulsants were the most commonly prescribed.

The World Health Organization (WHO), analgesic ladder for cancer pain relief is estimated to resolve approximately $80 \%$ of the pain in treated patients [16]. However, the neuropathic pain component is often more difficult to treat and European clinical practice guidelines to treat neuropathic pain are usually based on evidence from studies including non-cancer patients [17]. The Spanish Society of Medical Oncology (SEOM) clinical guidelines for the treatment of cancer pain acknowledge that neuropathic pain is not managed exclusively with opioids. They recommend coanalgesic drugs (i.e. antidepressants and anticonvulsants) to control neuropathic pain, regardless of the cause and corticosteroids for pain caused by nerve compression [18].
In general, patients with neuropathic pain report substantially low levels of health-related quality of life (QoL), and neuropathic pain severity is associated with a negative health impact [19]. Chronic neuropathic pain in non-cancer related pain conditions is associated with substantial physical, social, and economic consequences, such as health care utilization, work absenteeism, and unemployment [20]. It also negatively impacts regular daily activities [20]. Responses to the QoL questions and scales in this study indicate that many aspects of daily functioning were limited in the CRNP participants. Over half of this population $(\sim 54 \%)$ indicated that their symptoms impacted their employment status and the ratings on the SDS scale indicated that these participants' symptoms negatively impacted work, family and social life. These data are 
consistent with previous evidence of physical and psychological impairment in cancer patients experiencing neuropathic pain [4]. While there was no direct comparison with non-CRNP participants in this trial (as this was not part of the pre-planned analysis), these data provide further support for the need to establish more effective methods to identify and relieve neuropathic pain in patients with cancer, even in out-patient settings.

The need to identify and effectively manage neuropathic pain in patients with cancer has been identified [5]. However, there is currently no gold standard for the diagnosis of neuropathic pain, other than clinical assessment based on evidence of a lesion or disease of the somatosensory system, primarily from the history of the patient and clinical examination [21]. As yet, screening instruments to help identify neuropathic pain including Leeds Assessment of Neuropathic Symptoms and Signs (LANSS) Pain Scale [22], Douleur Neuropathique 4 Questions (DN4) [8], Neuropathic Pain Questionnaire [23], Neuropathic Pain Symptom Inventory [24], and PainDETECT [11], have not been specifically validated in patients with cancer [25]. When the PainDETECT questionnaire was compared with clinical assessment using the Edmonton Classification System of Cancer Pain (ECS$\mathrm{CP}$ ), it was shown that the PainDETECT questionnaire detected CRNP with less sensitivity and specificity than in non-cancer neuropathic pain [26]. This suggests that the PainDETECT questionnaire may require some adaptation to be a reliable assessment tool with high sensitivity for detecting CRNP.

Although the estimated prevalence of CRNP was similar before and after the use of the PainDETECT questionnaire, there were some shifts in physicians' opinion following the use of this tool, especially for participants with low end scores $(<13)$. Of interest, physicians indicated that the PainDETECT questionnaire did not help them evaluate whether the patient had CRNP for the majority $(60 \%)$ of participants, yet 18 out of 22 of the 'surveyed physician' population indicated that they found the PainDETECT questionnaire useful. While this may seem contradictory, the responses may provide insight regarding when this tool may be helpful to physicians. For example, physicians may only consult the PainDETECT scores in particular cases, which may introduce a risk of some CRNP cases being missed. As with the results of the main study, there were shifts in the numbers of participants with or without a diagnosis of CRNP, particularly for participants who received an initial diagnosis of 'unknown'. This may indicate that the questionnaire was not considered useful when physicians were confident about their initial diagnosis, but was considered useful in cases for which the diagnosis was less clear. It should be noted that PainDETECT was not designed to replace clinical assessment of neuropathic pain, but to indicate the potential likelihood that neuropathic pain is present [11].

This study is limited by missing and incomplete CRFs and was not designed as a comparative study. The results cannot therefore be generalized to the population of cancer patients as a whole, but do help to identify the need for reliable tools to help physicians effectively identify and manage neuropathic pain within out-patients with cancer.

\section{CONCLUSIONS}

The results of this sub-analysis of data collected from cancer patients visiting out-patient units in Spain are largely consistent with the results of the main study. The prevalence of CRNP within this out-patient population in Spain highlight the need for national guidelines to treat neuropathic pain, and in particular, cancer-related pain with a neuropathic component, particularly as there is evidence that the symptoms contribute to a negative impact on the patients QoL.

\section{CONFLICT OF INTEREST}

C. Garzón-Rodríguez has disclosed acting as a consultant/advisor for Pfizer. G. Atkinson, V. López-Gómez, M. Pérez Páramo and C. van Litsenburg are employees of Pfizer, own Pfizer Stock. G. Atkinson, M Pérez Páramo, and C. van Litsenburg have Pfizer stock options. L. Olay Gayoso, J. M. Sepulveda, E. Martínez, I. Peláez, and L. de la Cruz Merino have declared no conflict of interest.

\section{ACKNOWLEDGEMENT}

This study was funded by Pfizer. Medical writing support for the development of this manuscript was provided by Brenda Meyer, PhD, of Engage Scientific Solutions and was funded by Pfizer. The authors would like to thank Sarah Bowen for her role in the statistical analysis and all the Spanish investigators for their contribution to the study. The authors were not compensated for their work on the manuscript.
ABBREVIATIONS
CI
$=$ Confidence interval
CRF
$=$ Case report form
CRNP $=$ Cancer-related neuropathic pain
DN4 = Douleur Neuropathique 4
EuroQoL $=$ EQ-5D Health Questionnaire
$\mathrm{m}-\mathrm{BPI}-\mathrm{sF}=$ Modified Brief Pain Inventory Short Form
NICE $=$ National Institute for Health and Clinical Excellence
QoL $=$ Quality of life
SDS $\quad=$ Sheehan Disability Scale

\section{REFERENCES}

[1] Ferlay J, Parkin DM, Steliarova-Foucher E. Estimates of cancer incidence and mortality in Europe in 2008. Eur J Cancer 2010; 46(4): 765-81.

[2] Van den Beuken-van Everdingen MH, de Rijke JM, Kessels AG, Schouten HC, van Kleef M, Patijn J. Prevalence of pain in patients with cancer: a systematic review of the past 40 years. Ann Oncol 2007; 18: 1437-49.

[3] Baron R. Mechanisms of disease: neuropathic pain-a clinical perspective. Nat Clin Prac Neurol 2006; 2(2): 95-106.

[4] Tofthagen CS, McMillan SC. Pain, neuropathic symptoms, and physical and mental well-being in persons with cancer. Cancer Nurs 2010; 33(6): 436-44.

[5] Bennett MI, Rayment C, Hjermstad M, Aass N, Caraceni A, Kaasa S. Prevalence and aetiology of neuropathic pain in cancer patients: a systematic review. Pain 2012; 153(2): 359-65. 
[6] Manas A, Monroy JL, Ramos AA, et al. Prevalence of neuropathic pain in radiotherapy oncology units. Int $\mathrm{J}$ Radiat Oncol Biol Phys 2011; 81(2): 511-20.

[7] Garcia de Paredes ML, del Moral González F, Martínez del Prado $\mathrm{P}$, et al. First evidence of oncologic neuropathic pain prevalence after screening 8615 cancer patients. Results of the On study. Ann Oncol 2011; 22(4): 924-30.

[8] Bouhassira D, Attal N, Alchaar H, et al. Comparison of pain syndromes associated with nervous or somatic lesions and development of a new neuropathic pain diagnostic questionnaire (DN4). Pain 2005; 114(1-2): 29-36.

[9] Bennett MI, Attal N, Backonja MM, et al. Using screening tools to identify neuropathic pain. Pain 2007; 127(3): 199-203.

[10] Garzón-Rodríguez C, Lyras L, Olay Gayoso L, et al. Cancerrelated neuropathic pain in out-patient oncology clinics: a European survey. BMC Palliat Care 2013; 12(1): 41.

[11] Freynhagen R, Baron R, Gockel U, Tolle TR. painDETECT: a new screening questionnaire to identify neuropathic components in patients with back pain. Curr Med Res Opin 2006; 22(10): 191120.

[12] De Andrés J, Pérez-Cajaraville J, Lopez-Alarcón MD, et al. Cultural adaptation and validation of the painDETECT scale into Spanish. Clin J Pain 2012; 28(3): 243-53.

[13] Mendoza TR, Chen C, Brugger A, et al. The utility and validity of the modified brief pain inventory in a multiple-dose postoperative analgesic trial. Clin J Pain 2004; 20(5): 357-62.

[14] The EuroQol Group. EuroQol-a new facility for the measurement of health-related quality of life. The EuroQol Group. Health Policy 1990; 16(3): 199-208.

[15] Cleeland CS, Farrar JT, Hausheer FH. Assessment of cancerrelated neuropathy and neuropathic pain. Oncologist 2010; 15 Suppl 2: 13-8.

[16] Laird B, Colvin L, Fallon M. Management of cancer pain: basic principles and neuropathic cancer pain. Eur J Cancer 2008; 44(8): 1078-82.
[17] Piano V, Verhagen S, Schalkwijk A, et al. Treatment for Neuropathic Pain in Patients with Cancer: Comparative Analysis of Recommendations in National Clinical Practice Guidelines from European Countries. Pain Pract 2013: doi: 10.1111/papr.12036.

[18] Virizuela JA, Escobar Y, Cassinello J, Borrega P, SEOM (Spanish Society of Clinical Oncology). Treatment of cancer pain: Spanish Society of Medical Oncology (SEOM) recommendations for clinical practice. Clin Transl Oncol 2012; 14(7): 499-504.

[19] Doth AH, Hansson PT, Jensen MP, Taylor RS. The burden of neuropathic pain: a systematic review and meta-analysis of health utilities. Pain 2010; 149(2): 338-44.

[20] Vinik A, Emir B, Cheung R, Whalen E. Relationship Between Pain Relief and Improvements in Patient Function/Quality of Life in Patients With Painful Diabetic Peripheral Neuropathy or Postherpetic Neuralgia Treated With Pregabalin. Clin Ther 2013: [Epub ahead of print].

[21] Treede RD, Jensen TS, Campbell JN, et al. Neuropathic pain: redefinition and a grading system for clinical and research purposes. Neurology 2008; 70(18): 1630-5.

[22] Bennett M. The LANSS Pain Scale: the Leeds assessment of neuropathic symptoms and signs. Pain 2001; 92(1-2): 147-57.

[23] Krause SJ, Backonja MM. Development of a neuropathic pain questionnaire. Clin J Pain 2003; 19(5): 306-14.

[24] Bouhassira D, Attal N, Fermanian J, et al. Development and validation of the Neuropathic Pain Symptom Inventory. Pain 2004; 108(3): 248-57.

[25] Piano V, Verhagen S, Schalkwijk A, et al. Diagnosing Neuropathic Pain in Patients with Cancer: Comparative Analysis of Recommendations in National Guidelines from European Countries. Pain Pract 2012: doi: 10.1111/papr.12018.

[26] Rayment C, Hjermstad MJ, Aass N, et al. Neuropathic cancer pain: Prevalence, severity, analgesics and impact from the European Palliative Care Research Collaborative-Computerised Symptom Assessment study. Palliat Med 2013; 27(8): 714-21.

(C) Garzón-Rodríguez et al.; Licensee Bentham Open.

This is an open access article licensed under the terms of the Creative Commons Attribution Non-Commercial License (http://creativecommons.org/licenses/by-nc/3.0/) which permits unrestricted, non-commercial use, distribution and reproduction in any medium, provided the work is properly cited. 